\title{
The magnetic strip(s) in the advanced phases of stellar evolution
}

\section{Theoretical convective turnover timescale and Rossby number for low- and intermediate-mass stars up to the AGB at various metallicities ${ }^{\star}$}

\author{
C. Charbonnel ${ }^{1,2}$, T. Decressin ${ }^{3}$, N. Lagarde ${ }^{4}$, F. Gallet ${ }^{1}$, A. Palacios ${ }^{5}$, M. Aurière ${ }^{6}$, R. Konstantinova-Antova ${ }^{7,2}$, \\ S. Mathis ${ }^{8}$, R. I. Anderson ${ }^{9, \star \star}$, and B. Dintrans ${ }^{2}$
}

1 Department of Astronomy, University of Geneva, Chemin des Maillettes 51, 1290 Versoix, Switzerland e-mail: Corinne.Charbonnel@unige.ch

2 IRAP, UMR 5277, CNRS and Université de Toulouse, 14 av. E. Belin, 31400 Toulouse, France

3 INAF-Osservatorio Astronomico di Roma, via di Frascati 33, 00040 Monteporzio, Italy

4 Institut Utinam, CNRS UMR 6213, Univ. Bourgogne Franche-Comté, OSU THETA Franche-Comté-Bourgogne, Observatoire de Besançon, BP 1615, 25010 Besançon Cedex, France

5 LUPM, UMR5 299, Université de Montpellier, CNRS, place Eugène Bataillon, 34095 Montpellier, France

6 IRAP, UMR 5277, CNRS and Université de Toulouse, 57 av. d'Azereix, BP 826, 65008 Tarbes Cedex, France

7 Institute of Astronomy and NAO, Bulgarian Academy of Sciences, 72 Tsarigradsko shose, 1784 Sofia, Bulgaria

${ }^{8}$ Laboratoire AIM Paris-Saclay, CEA/Irfu Université Paris-Diderot CNRS/INSU, 91191 Gif-sur-Yvette, France

9 Physics and Astronomy Department, Johns Hopkins University, Baltimore, MD 21218, USA

Received 12 June 2015 / Accepted 23 March 2017

\begin{abstract}
Context. Recent spectropolarimetric observations of otherwise ordinary (in terms e.g. of surface rotation and chemical properties) G, $\mathrm{K}$, and $\mathrm{M}$ giants have revealed localized magnetic strips in the Hertzsprung-Russell diagram coincident with the regions where the first dredge-up and core helium burning occur.

Aims. We seek to understand the origin of magnetic fields in such late-type giant stars, which is currently unexplained. In analogy with late-type dwarf stars, we focus primarily on parameters known to influence the generation of magnetic fields in the outer convective envelope.

Methods. We compute the classical dynamo parameters along the evolutionary tracks of low- and intermediate-mass stars at various metallicities using stellar models that have been extensively tested by spectroscopic and asteroseismic observations. Specifically, these include convective turnover timescales and convective Rossby numbers, computed from the pre-main sequence (PMS) to the tip of the red giant branch (RGB) or the early asymptotic giant branch (AGB) phase. To investigate the effects of the very extended outer convective envelope, we compute these parameters both for the entire convective envelope and locally, that is, at different depths within the envelope. We also compute the turnover timescales and corresponding Rossby numbers for the convective cores of intermediate-mass stars on the main sequence.

Results. Our models show that the Rossby number of the convective envelope becomes lower than unity in the well-delimited locations of the Hertzsprung-Russell diagram where magnetic fields have indeed been detected.

Conclusions. We show that $\alpha-\Omega$ dynamo processes might not be continuously operating, but that they are favored in the stellar convective envelope at two specific moments along the evolution tracks, that is, during the first dredge-up at the base of the RGB and during central helium burning in the helium-burning phase and early-AGB. This general behavior can explain the so-called magnetic strips recently discovered by dedicated spectropolarimetric surveys of evolved stars.
\end{abstract}

Key words. stars: activity - stars: interiors - stars: magnetic field - stars: rotation - dynamo

\section{Introduction}

Magnetic fields are actively searched for at the surface of all kinds of stars throughout the Hertzsprung-Russell diagram (HRD), as they probably impact stellar evolution from birth to death in various ways (see e.g., Donati \& Landstreet 2009 for a general review; and Wade et al. 2016; Alecian et al. 2013; Vidotto et al. 2014; Folsom et al. 2016 for recent spectropolarimetric surveys on massive, intermediate-mass, and low-mass

\footnotetext{
* The grid of models is only available at the CDS

via anonymous ftp to cdsarc.u-strasbg. fr (130.79.128.5) or via http://cdsarc.u-strasbg.fr/viz-bin/qcat?]/A+A/605/A102 $\star \star$ Swiss National Science Foundation Fellow.
}

stars). Recently, magnetic fields have been unambiguously detected via Zeeman signatures in a large sample of single G-K giants observed with the spectropolatimeters TBL/Narval and CFHT/ESPaDOnS (Konstantinova-Antova et al. 2013; Aurière et al. 2015; Borisova et al. 2016; Tsvetkova et al. 2017). Interestingly, the cool intermediate-mass evolved stars with surface magnetic fields are found to cluster in certain regions of the HRD that correspond to precise moments of their evolution where convective envelopes make up a significant fraction of the stellar mass. These observations are of particular importance for stellar-evolution modeling, since magnetic fields may play a crucial role in the angular momentum evolution of different types of stars. For instance, it is well established that magnetic 
fields affect the rotation rate of solar-type stars through the torque applied to stellar surfaces by magnetically coupled stellar winds (e.g., Schatzman 1962; Matt et al. 2015; Réville et al. 2015; Amard et al. 2016). However, the efficiency and the impact of magnetic braking for evolved stars are not well studied yet. In addition, because of the angular momentum they transport (e.g., Spruit 1999; Mathis \& Zahn 2005), magnetic fields may play an important role in explaining the properties of core and surface rotation in giants as seen by asteroseismology (e.g., Beck et al. 2012; Mosser et al. 2012b,a; Deheuvels et al. 2012, 2014; Cantiello et al. 2014; Di Mauro et al. 2016), as well as the rotation rate of white dwarf remnants (Suijs et al. 2008). By comparing the observed rotational and magnetic properties of stars at different evolutionary phases with the predictions of rotating stellar models, one may thus obtain strong constraints on the input physics of the stellar models. Conversely, it is important to know whether the possible presence and the global properties of the magnetic field of a given star can be anticipated from its position in the Hertzsprung-Russell diagram (see Gregory et al. 2012 for the case of pre-main sequence stars).

In main sequence solar-like stars and their red giant descendants, dynamo processes are invoked to generate magnetic fields through turbulence and rotational shear within the stellar convective envelopes or within a thin shear layer at the interface between the convective and the radiative regions (so-called tachocline; see e.g., Charbonneau 2014, for a review and references therein). This is supported by the existence of a tight correlation between the measured magnetic field strength and the rotational properties (namely the rotational period and the convective Rossby number Ro, hereafter Rossby number for simplicity) of magnetic red giant stars with known rotational periods (Aurière et al. 2015). In hotter, more massive main sequence and Ap stars that do not have extended convective envelopes, fossil magnetic fields are generally invoked to explain magnetic field properties (e.g., Neiner et al. 2015). Moreover, those early-type stars might produce internal magnetic fields in their vigorous convective core during the central hydrogen-burning phase (e.g., Brun et al. 2005; Stello et al. 2016). When these objects move towards the red giant branch (RGB), deeply buried magnetic fields might survive and interact with the deepening convective envelope. Some post-main sequence stars have been recently shown to be possible Ap star descendants (see e.g., Aurière et al. 2014, and references therein). However, the details on how dynamos and field dredge-up can operate in giant stars are not yet sufficiently understood.

The properties of the convective regions as well as the rotation velocity of stars depend on their initial mass and metallicity, and strongly vary along their evolution. As a consequence, the efficiency of the dynamo-driven mechanisms is expected to vary as stars of various initial masses evolve. Therefore, it is necessary to investigate how the main stellar quantities that are currently used to evaluate the efficiency of magnetic field generation in stellar interiors do change as stars of different initial masses evolve. In this context, the classical relevant quantities are the convective turnover timescale, which varies with spectral type as a function of the evolving properties of the stellar convective zones, and the Rossby number, which quantifies the interactions between convection and rotation. This latest quantity is proportional to the ratio between the stellar rotation period and the convective turnover time, and it describes how the Coriolis force affects convective eddies. Low Rossby values (of the order or below unity) are expected in the case of stars with sufficiently fast rotation for efficient magnetic field generation (e.g., Durney \& Latour 1978; Mangeney \& Praderie 1984; Noyes et al. 1984; Brun et al. 2015; Augustson et al. 2016).

The present paper describes how these classical dynamo parameters vary throughout the evolution of low- and intermediatemass stars at various metallicities, based on the predictions on one-dimensional (1D) stellar models. This allows us to identify the evolution phases during which stellar dynamo may operate and to motivate further studies involving detailed, sophisticated, and numerically expensive, magnetohydrodynamic simulations that are needed to capture all the processes involved in global stellar dynamos (e.g., Brun 2015, and references therein). Here we use the grid of stellar models presented in Lagarde et al. (2012), whose characteristics are briefly recalled in Sect. 2; we also describe the content of the electronic tables. In Sect. 3, we present convective turnover timescales from the pre-main sequence (PMS) to the early asymptotic giant phase (AGB) and along the thermally pulsing AGB (TP-AGB) for selected cases, for the convective envelope of stars with initial masses between 0.85 and $6.0 M_{\odot}$, and for two metallicities $Z=0.014$ and $Z=0.0001$. We discuss how the turnover timescale varies with depth inside the stellar convective envelope as stars evolve, and explore how this depends on stellar mass and metallicity. In Sect. 4 we give the theoretical Rossby numbers for our stellar models including rotation and discuss their variations from the zero-age main sequence (ZAMS) to the early-AGB. We present theoretical evidence for the existence of two magnetic strips that low- and intermediate-mass stars are predicted to cross in the advanced phases of their evolution. In Sect. 5 we compare our model predictions with the position in the HRD of red giant stars for which surface magnetic fields have been detected. We summarize our results in Sect. 6. In the Appendix, we provide the turnover timescales and the Rossby numbers for the convective core of intermediate-mass main sequence stars for the solar metallicity grid.

\section{Reference grid of stellar models and content of the electronic tables}

This paper is based on the grid of standard and rotating stellar models computed by Lagarde et al. $(2012)^{1}$ with the code STAREVOL (V3.00) for a range of initial masses between 0.85 and $6 M_{\odot}$ and for four values of $[\mathrm{Fe} / \mathrm{H}]=0,-0.56,-0.86$, -2.16 (corresponding respectively to metallicities $Z=0.014^{2}$, $4 \times 10^{-3}, 2 \times 10^{-3}$, and $\left.10^{-4}\right)$. Convection is treated according to the classical mixing length formalism with $\alpha_{\mathrm{MLT}}=1.6$. The boundary between convective and radiative layers is defined with the Schwarzschild criterion. An overshoot parameter $\mathrm{d} / \mathrm{H}_{p}$ is taken into account for the convective core; it is set to 0.05 for stars with less than $2.0 M_{\odot}$ and to 0.10 for more massive stars. The evolution of angular momentum within the radiative stellar regions is computed using the complete formalism developed by Zahn (1992), Maeder \& Zahn (1998), and Mathis \& Zahn (2004), which takes into account advection by meridional circulation and diffusion by shear turbulence (see Palacios et al. 2003, 2006; Decressin et al. 2009). Convective regions are treated as solid bodies. The impact of rotation-induced mixing and thermohaline instability on the global parameters and the chemical properties of low- and intermediate-mass stars is discussed

\footnotetext{
http://cdsarc.u-strasbg.fr/viz-bin/qcat?]/A+A/543/ A 108

${ }^{2} Z=0.014$ corresponds to $Z_{\odot}$, with Asplund et al. (2005) chemical composition except for $\mathrm{Ne}$ for which we use the value derived by Cunha et al. (2006)
} 
in Charbonnel \& Lagarde (2010). The corresponding nucleosynthetic yields are presented in Lagarde et al. (2011). The relevant classical stellar parameters as well as the theoretical global asteroseismic properties of the models can be found in Lagarde et al. (2012).

The initial rotation velocity of the models corresponds to $30 \%$ of the critical velocity at the ZAMS (Lagarde et al. 2014). For the $2 M_{\odot}, Z_{\odot}$ case, three additional rotating models are presented here, with initial rotation velocity on the ZAMS of 50, 163 , and $250 \mathrm{~km} \mathrm{~s}^{-1}$, which correspond to 15,44 , and $68 \%$ of the critical velocity. Magnetic braking is included following Kawaler (1988) from the ZAMS onward for all low-mass models, that is, with $1.25 M_{\odot}, Z_{\odot}$, and $0.85 M_{\odot}, Z=0.0001$. For more massive stars that have very thin convective envelope on the main sequence, the evolution of the surface velocity is governed by secular effects and internal transport of angular momentum through meridional circulation and shear turbulence. Rotation is accounted for throughout the evolution from the ZAMS up to the beginning of the TP-AGB phase for all the models that ignite central helium burning in non-degenerate conditions; for the less massive models, rotation is included only until the tip of the red giant branch.

We provide electronic files containing sets of relevant physical quantities tabulated for 500 evolution points along the evolution tracks from the PMS up to the early-AGB phase (see Sect. 3.1 in Lagarde et al. 2012). For each model, we give the turnover timescales at different heights in the convective envelope $\tau_{H_{p} / 2}, \tau_{H_{p}}, \tau_{R_{\mathrm{EC}} / 2}, \tau_{M_{\mathrm{EC}} / 2}$, and $\tau_{\max }$, as well as the corresponding Rossby numbers (see Sects. 3 and 4 ) in addition to the quantities already listed in Table 2 of Lagarde et al. (2012).

\section{Convective turnover timescales}

\subsection{Definitions}

The local convective turnover time at a given radius $r$ inside the convective envelope, $\tau_{\mathrm{c}}(r)$ and the so-called global convective turnover time, $\tau_{\mathrm{g}}$ are defined as in Gilliland (1985)

$\tau_{\mathrm{c}}(r)=\alpha_{\mathrm{MLT}} H_{P}(r) / V_{\mathrm{c}}(r)$,

and

$\tau_{\mathrm{g}}=\int_{R_{\mathrm{b}}}^{R_{\mathrm{t}}} \frac{\mathrm{d} r}{V_{\mathrm{c}}(r)}$.

$H_{P}(r)$ is the local pressure scale height, $\alpha_{\text {MLT }}$ is the mixinglength parameter, $R_{\mathrm{b}}$ and $R_{\mathrm{t}}$ are the radii at the bottom and at the top of the convective envelope, respectively (taken where $\nabla-\nabla_{a d}=0$, following Schwarzschild criterion). According to the mixing length theory the average local convective velocity is defined as

$V_{\mathrm{c}}^{2}(r)=g \delta\left(\nabla-\nabla_{a d}\right) \frac{\alpha_{\mathrm{MLT}}^{2}}{8 H_{P}(r)}$,

where $g$ is gravity and $\delta=\partial \ln \rho / \partial \ln T$ is determined from the equation of state.

Since $\tau_{\mathrm{c}}(r)$ is strongly depth-dependent (see Sect. 3.2.1), and since it is not well established where exactly a dynamo operates within the stellar convective envelope, we determine its value at different depths within the stellar convective envelope. $\tau_{H_{p} / 2}$ and $\tau_{H_{p}}$ are the convective turnover times at half a pressure scale height and at a pressure scale height above the base of the convective envelope, respectively. $\tau_{R_{\mathrm{EC}} / 2}$ and $\tau_{M_{\mathrm{EC}} / 2}$ are computed at

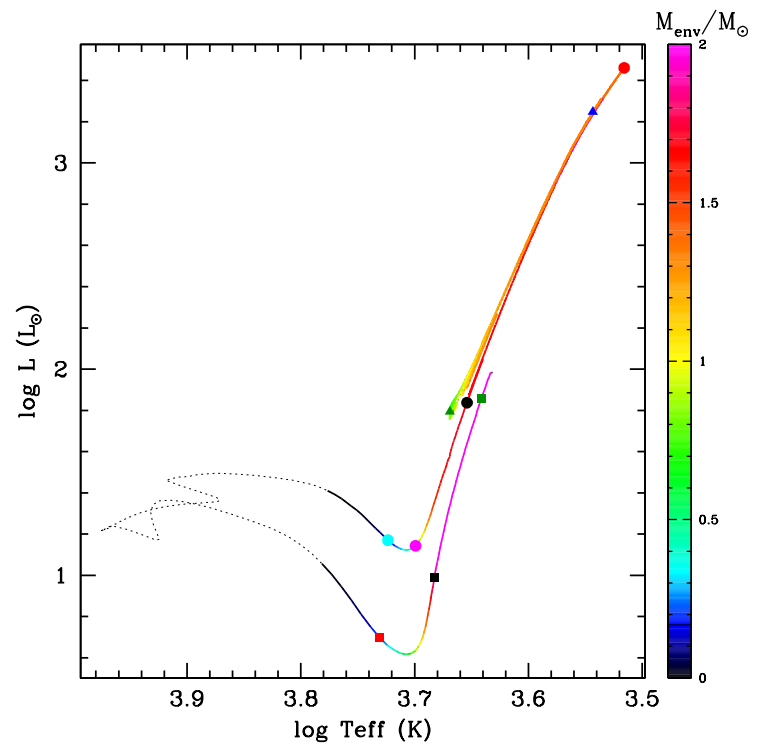

Fig. 1. Variations of the size of the convective envelope (in solar mass, color-coded) along the track of the $2 M_{\odot}, Z_{\odot}$ standard model. The dotted line corresponds to the phases when the convective envelope contains less than $0.001 M_{\odot}$. Specific evolution points are selected. On the PMS, the green, black, and red squares correspond respectively to stages when the star is still fully convective, and when the mass of the receding convective envelope is 1.9 and $0.26 M_{\odot}$. In the advanced phases, the magenta, cyan, black circles correspond respectively to the beginning, middle, and end of the first dredge-up, that is, when the mass of the convective envelope is $0.13,0.87$, and $1.7 M_{\odot}$. Red circle, green and blue triangles correspond to the RGB tip, to the middle of central helium-burning, and to the end of the second dredge-up on the earlyAGB, respectively; at these evolutionary points the mass of the convective envelope is respectively $1.39,0.7$, and $1.31 M_{\odot}$.

half of the radius and half of the mass of the convective envelope, and $\tau_{\max }$ is the maximum convective turnover time within the convective envelope. We investigate below how these quantities vary from the PMS along the Hayashi track until the end of the early-AGB for all models included in the grid that comprises both standard models and models including rotation, for four different metallicities. We provide a detailed discussion of the $2 M_{\odot}, Z_{\odot}$ standard model in Sect. 3.2.1 and then describe the trends with mass and metallicity in Sect. 3.2.3. We also compute the turnover timescales in the convective core of solar metallicity main sequence stars (when this core is present), using the same definitions as above, and present the results in the Appendix. This allows us to provide complete diagnoses for the studied stars.

\subsection{Turnover timescale in stellar convective envelopes along the evolution}

\subsubsection{The case of the $2 M_{\odot}, Z_{\odot}$ model}

Figure 1 shows the variations of the mass enclosed in the convective envelope along the evolution track in the HertzsprungRussell diagram in the case of the $2 M_{\odot}, Z_{\odot}$ standard model. We select evolution points along the track for which we show in Fig. 2 the profiles of the various quantities that enter in the definition of the turnover timescale. Finally, Fig. 3 shows the variation of the convective turnover timescales at different depths within 


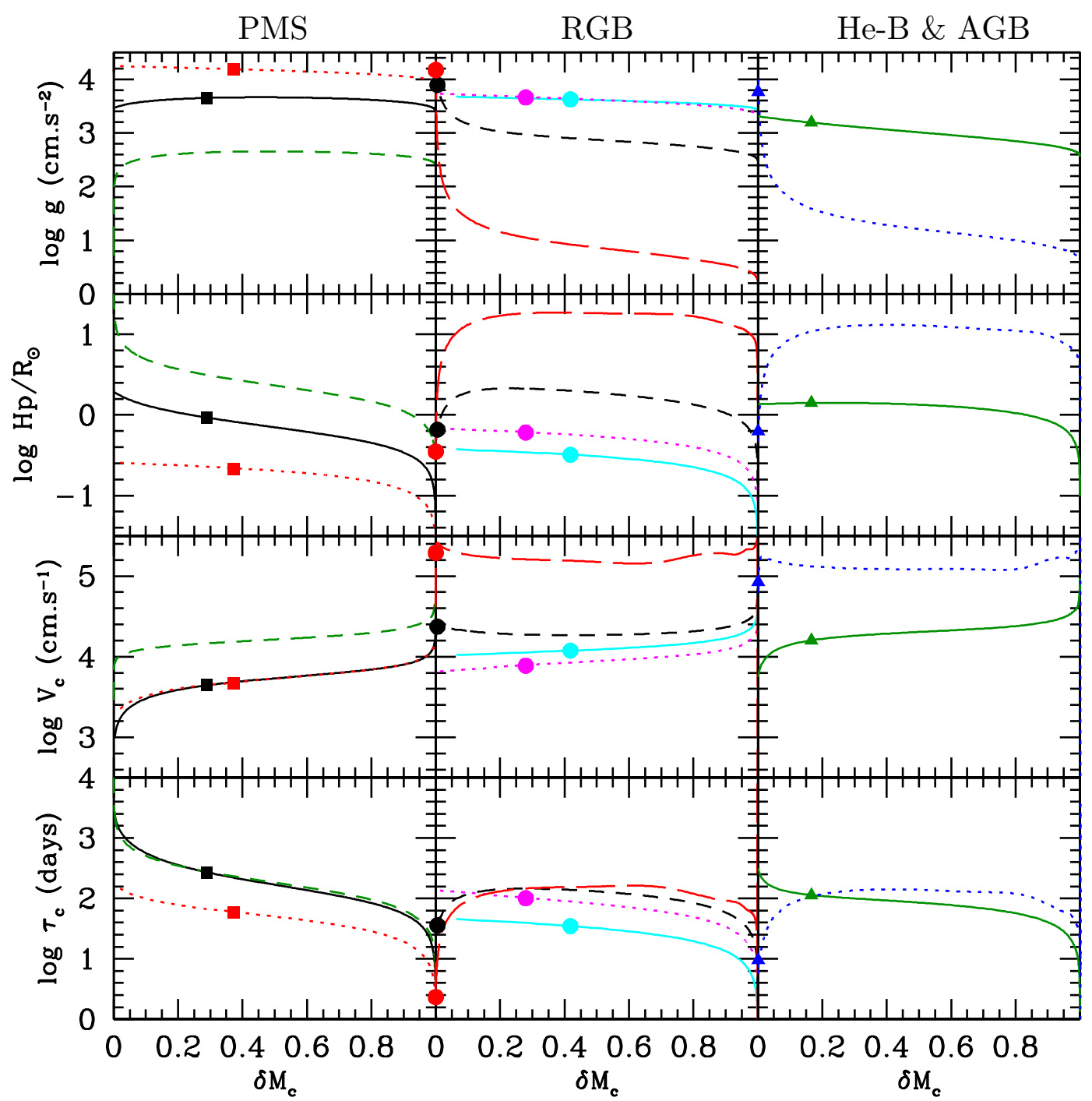

Fig. 2. Variations within the convective envelope of gravity, pressure scale height, convective velocity, and turnover time (from top to bottom) for the $2 M_{\odot}, Z_{\odot}$ standard model. The abscissa is the scaled mass coordinate $\delta M_{\text {conv }}\left(\delta M_{\text {conv }}=0\right.$ and 1 respectively at the base and at the top of the convective envelope). The colors correspond to evolution points selected in Fig. 1 (see there for the corresponding values of the envelope thickness). PMS, RGB, and advanced phases are shown on the left, middle, and right panels, respectively. The corresponding colored symbols indicate the location of $H_{p} / 2$ above the base of the convective envelope (for the full convective model on the PMS - green line - this point would be located outside the star, at a radius of $\sim 26 R_{*}$ ).

the convective envelope $\left(\tau_{H_{p} / 2}, \tau_{H_{p}}, \tau_{R_{\mathrm{EC}} / 2}, \tau_{M_{\mathrm{EC}} / 2}, \tau_{\mathrm{max}}\right.$, and $\tau_{\mathrm{g}}$, in days, logarithmic scale) throughout the evolution of the star.

Pre-main sequence - PMS evolution proceeds along increasing $T_{\text {eff }}$. At first, the star is fully convective. As it contracts along the Hayashi track, the convective envelope quickly decreases in mass. Gravity and $H_{p}$ strongly vary (increase and decrease, respectively), which impacts $V_{\text {c }}$ following Eq. (3) (Fig. 2). Consequently the convective turnover timescale decreases during the contraction phase. Then the base of the convective envelope as well as the various $\tau_{\mathrm{c}}(r)$ stay relatively constant as the star evolves towards the ZAMS. This behavior has already been described in the literature (cf. e.g., Kim \& Demarque 1996 and Landin et al. 2010). In our $2 M_{\odot}, Z_{\odot}, \tau_{H_{p} / 2}$ (respectively $\tau_{\max }$ ) drops from 290 to 0.04 days (respectively from $3150 \mathrm{yr}$ to 0.17 days) along the PMS.

Main sequence - while on the MS, the external convective region of this model is extremely thin (less than $0.001 M_{\odot}$ ), and it varies very little in mass. Therefore the various $\tau_{\mathrm{c}}(r)$ have very low values, and they stay relatively constant as the star evolves towards cooler $T_{\text {eff }}$ from the ZAMS to the Terminal Age Main Sequence (TAMS).

Hertzsprung gap and $R G B$ - as the star adjusts to hydrogenshell burning and moves towards cooler $T_{\text {eff }}$ along the subgiant branch (also called Hertzsprung gap), the convective envelope strongly deepens, thereby increasing in mass (the so-called first dredge-up, hereafter 1DUP), before receding again as the star expands in radius along the RGB (see Fig. 1). Simultaneously, within the bulk of the convective envelope the pressure scale height strongly increases while gravity decreases by approximately three orders of magnitude up to the RGB tip (Fig. 2). As a consequence, the turnover timescale strongly rises at all depths within the convective envelope during the 1DUP. In the upper part of the RGB (Fig. 3), $\tau_{H_{p} / 2}$ and $\tau_{H_{p}}$ slightly decrease again, due to the pressure profile, whose gradient then strongly steepens close to the base of the convective envelope. The other $\tau_{\mathrm{c}}$ all continue to increase up to the RGB tip mainly due to the 
C. Charbonnel et al.: The magnetic strip(s) in the advanced phases of stellar evolution
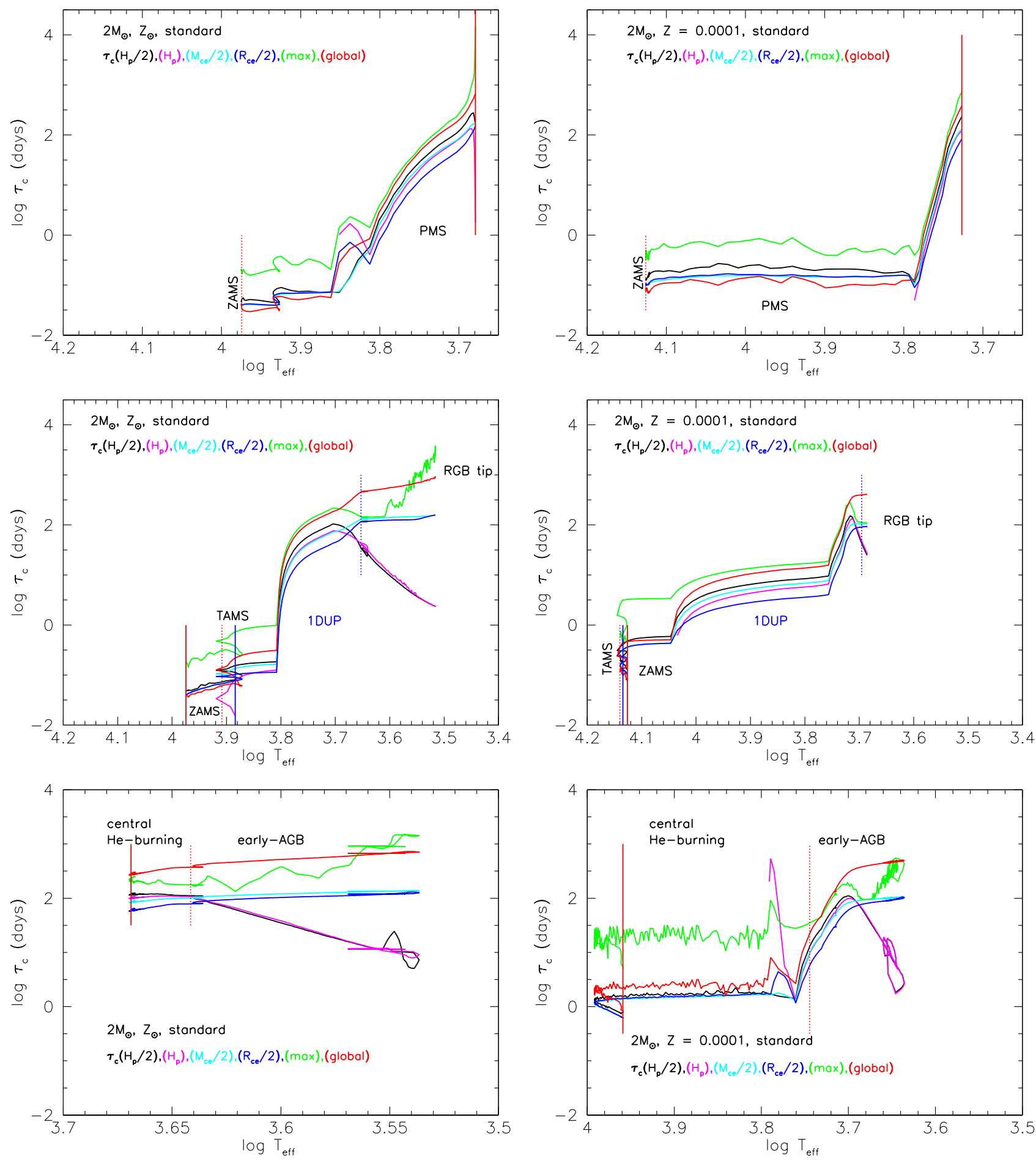

Fig. 3. Evolution of the convective turnover time at different depths within the convective envelope as a function of effective temperature on the PMS (top), from the ZAMS up to the RGB tip (middle), and from the beginning of central helium-burning up to the end of the early-AGB (bottom). The vertical lines indicate the beginning and the end (solid and dashed, respectively) of the PMS, of the central H- and He-burning phases, and of the first dredge-up (in blue). Left and right figures correspond to the $2 M_{\odot}$ standard models computed at $Z_{\odot}$ and $Z=0.0001$ respectively. The magenta lines that are associated to $\tau_{\mathrm{c}}\left(H_{p}\right)$ are sometimes interrupted; this corresponds to evolution phases when the radius at a pressure scale height $H_{p}$ above the base of the convective envelope becomes larger than the radius of the star.

decrease in gravity. The increase in the value of $\tau_{\max }$ in the upper part of the RGB is driven by the changes in opacity in the stellar atmosphere that weaken the convection velocity near the surface.
Clump and early-AGB - when the star ignites central helium burning in the so-called clump, its convective envelope is much thinner than at the RGB tip, and gravity has increased again; the quantities associated to the different definitions of $\tau_{\mathrm{c}}$ then stay 
relatively constant. As the star evolves towards the early-AGB, its convective envelope deepens again. During this phase, $\tau_{\mathrm{c}}$ increase again within the bulk of the convective envelope, except in the deepest convective layers where the gradient of $H_{P}$ strongly steepens.

\subsubsection{Metallicity effect}

The question of whether one can use the same set of convective turnover times for stars of different populations was addressed by Rucinski \& Vandenberg (1990). However, their study was limited to the case of zero-age main-sequence solar-type stars with $[\mathrm{Fe} / \mathrm{H}]$ above solar. Using our model grid, we can estimate the impact of large variations in metallicity on the convective turnover timescale over a wide range of evolutionary phases (from the PMS to the AGB).

A low-mass stellar model at $Z=0.0001$ evolves at higher luminosity than its $Z_{\odot}$ counterpart, and it reaches higher $T_{\text {eff }}$ on the ZAMS. This results from the well-known impact of metals on the radiative opacity and on the mean molecular weight in stellar interiors (e.g., Kippenhahn et al. 2012). This slightly modifies the convective properties of the star, leading to higher values of $\tau_{\mathrm{c}}(r)$ at a given effective temperature during the evolution towards the ZAMS, as shown in Fig. 3 for the $2 M_{\odot}$ case. For the same reason (i.e. opacity effects), the different $\tau_{\mathrm{c}}(r)$ are also higher for the most metal-poor model on the MS, the subgiant phase, the RGB, and the AGB. During central helium-burning, the low-metallicity model evolves along so-called blue loops, reaching much higher $T_{\text {eff }}$ than the $Z_{\odot}$ model, which stays in the red clump. This results in thinner convective envelope and lower $\tau_{\mathrm{c}}$ at all depths. Consequently, we can conclude that sets of convective turnover times computed with the relevant metallicity should be used for stars of different populations.

\subsubsection{Mass dependence}

The general behavior of the convective turnover timescales at different depths within the stellar convective envelope along the evolution tracks is similar for all stars to that described above for the $2 M_{\odot}$ case. To illustrate the evolutionary aspects we chose to focus on $\tau_{H_{p} / 2}$ which is shown in Fig. 4 for all the solar metallicity models of our grid along the PMS and from the ZAMS onwards (top and bottom panels respectively). Importantly, we see that for all the models, $\tau_{H_{p} / 2}$ sharply increases when the postmain sequence stars reach the middle of the Hertzsprung gap. In particular, $\tau_{H_{p} / 2}$ becomes higher than approximately one day when $\log T_{\text {eff }}$ becomes lower than $\sim 3.8-3.7$ (see also Fig. 3), which corresponds to the evolutionary point when the deepening convective envelope reaches a mass of $\sim 1$ to $5 \%$ of the stellar mass. It is maximum (with very similar values over the whole mass range considered here) at the base of the RGB, when the convective envelope encompasses $\sim 40 \%$ of the total stellar mass. Interestingly, the domain in effective temperature when the convective turnover times are the highest is very similar on the pre- and post-main sequences, as the fractional envelope mass $M_{\mathrm{env}} / M_{*}$ of the pre- and post-main sequence stars are very similar at a given effective temperature. This proxy for internal structure is a direct reflection of the growth of the convective envelope in the stellar evolution phases when rapid stellar contraction or extension occur (pre- and post-main sequence respectively). Gregory et al. (2012) suggested that it can be used as a proxy for the general characteristics of a magnetic field in
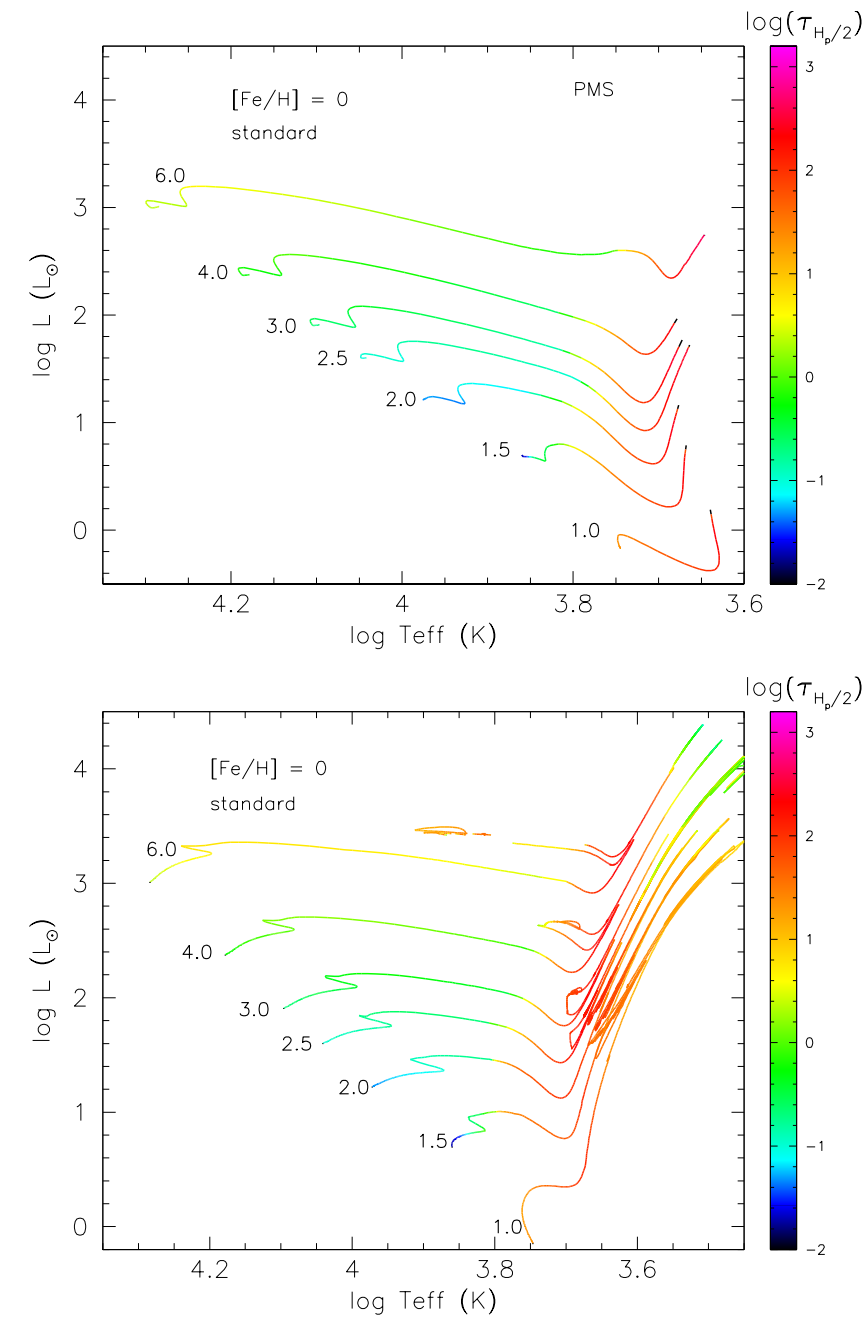

Fig. 4. Color-coded convective turnover time $\tau_{H_{p} / 2}$ (in logarithmic scale) in the stellar convective envelope along the PMS and beyond the ZAMS (top and bottom panels respectively) for the solar metallicity models. Initial stellar masses (in solar mass) are indicated on the tracks.

PMS stars at a given position in the HRD. Here, we anticipate that it is also the case for post-main sequence stars.

\subsubsection{Impact of rotation}

We refer to Charbonnel \& Lagarde (2010) and Lagarde et al. (2012) for a detailed discussion of the impact of rotation on the global parameters of our grid of models, and focus only on the impact on the evolution paths in the HRD that are relevant for the convective properties of the stars. For a given stellar mass and metallicity, the evolutionary effects of rotation shift the evolution tracks to slightly higher luminosity. However, for a given $T_{\text {eff }}$ the properties of the convective envelope change very little, and one finds very similar $\tau_{H_{p} / 2}$ values (the same is true for all $\left.\tau_{\mathrm{c}}(r)\right)$. Therefore, considering our current modeling of the impact of rotation on stellar evolution one can conclude that for a given stellar mass the turnover timescales do not significantly depend on initial rotation velocity.

\section{Rossby number}

\subsection{Definition}

The Rossby number Ro was first defined by Rossby (1939) as the ratio of advection to the Coriolis acceleration. The advective 
term can be expressed as $\sim U^{2} / L$ (which gives the amplitude of $(\boldsymbol{u} \cdot \nabla) \boldsymbol{u})$ where $U$ and $L$ are the characteristic speed and length scale of the system, respectively, and the Coriolis term is $\sim U \Omega$, with $\Omega$ representing the angular velocity of the star. The Rossby number is thus given by

$R o \sim \frac{U^{2}}{L} \frac{1}{\Omega U}=\frac{U}{L \Omega}$.

In the case of a star, the ratio $U / L$ is the characteristic flow timescale of the stellar fluid, that is, the convective turnover timescale, and $1 / \Omega$ is, to a factor $2 \pi$, the characteristic period of the system, that is, the stellar rotation rate $\left(P_{\text {rot }}=2 \pi / \Omega\right)$, which leads to

$R o=\frac{P_{\text {rot }}}{\tau_{\mathrm{c}}}$.

A system that is strongly affected by Coriolis forces will then have a small Rossby number, while a system in which inertial forces dominate will have a large Rossby number. Classically, dynamo action is expected to become more efficient when Ro is lower than 1 (e.g., Brun et al. 2015; Augustson et al. 2016, and references therein). Indeed, when the Rossby number is small, convective flows are strongly influenced by the Coriolis acceleration. As a consequence, they become quasi-2D and they generate a stronger kinetic helicity coherent in space and in time. This kinetic helicity is a key actor to sustain an efficient $\alpha$-effect that generates a mean poloidal field thanks to the action of smallscale convective vorticies twisting the mean toroidal magnetic field. In addition, a low convective Rossby number supports a strong differential rotation (e.g., Brown et al. 2008; Varela et al. 2016), which strengthens the $\Omega$-effect where the differential rotation winds up the mean poloidal field into a mean toroidal magnetic field. Therefore, when the Rossby number decreases both $\alpha$ and $\Omega$ effects and the resulting dynamo action are expected to be stronger. This is also predicted thanks to new theoretical scaling laws giving magnetic energy as a function of the convective Rossby number (Augustson et al. 2017).

In the literature, the Rossby number is generally computed with the use of the turnover timescale at half a pressure scale height above the base of the convective envelope $\tau\left(H_{p} / 2\right)$, which we call $\operatorname{Ro}\left(H_{p} / 2\right)$. However, for giant stars that have deep and extended convective envelopes, it is not clear where the dynamo operates. Therefore, we also compute the various Rossby numbers for the different $\tau_{\mathrm{c}}(r)$ within the convective envelope, and we use

$P_{\text {rot }}=2 \pi \frac{R_{*}}{V_{\text {surf }}}$

with $V_{\text {surf }}$ the surface velocity of our rotating models. For core convection (Appendix), we compute Ro(core) using the global turnover timescale $\tau_{\mathrm{g} \text {,core }}$ and the mean angular velocity of the core.

\subsection{Rossby number in the convective envelope along the evolution and the theoretical magnetic strips}

\subsubsection{The $2 M_{\odot}, Z_{\odot}$ case}

Figure 5 shows the evolution of the rotational period for $2 M_{\odot}$, $Z_{\odot}$ models computed with different initial rotation velocities, from the ZAMS up to the tip of the RGB. Since no magnetic braking is applied to this model, the variations of $P_{\text {rot }}$ are driven by secular evolution for this mass range, that is, by the variations of the stellar radius $R_{*}$.
Figure 5 illustrates the ZAMS-to-RGB evolution of the Rossby number $\operatorname{Ro}\left(H_{p} / 2\right)$ for the $2 M_{\odot}, Z_{\odot}$ models computed with different initial rotation velocities. $\operatorname{Ro}\left(H_{p} / 2\right)$ drops when the convective envelope deepens in mass during the 1DUP. We obtain the same behavior for all Ro calculated with the different convective timescale definitions. For all the initial rotation rates assumed here, the different Ro than unity, which is the classical value at which $\alpha-\Omega$ dynamos are expected to become stronger, although the $T_{\text {eff }}$ at which this occurs during the Hertzsprung gap depends on the initial rotation velocity (the higher the $V_{\text {ZAMS }}$, the earlier Ro reach values below unity). After they reach a minimum at the base of the RGB, all Ro quickly increase mainly due to the expansion of the envelope as the star climbs the RGB. It is interesting to note that for a given mass, Fig. 5 predicts that the width of the magnetic strip depends on the initial rotation rate (see Fig. 5 for the evolution of the rotation rates). The faster rotating models have $\log$ Ro $<0$ for a wider range of temperatures (respectively $\sim 1450$ and $700 \mathrm{~K}$ along the RGB phase for the faster and the slower rotation rates we assumed for the $2 M_{\odot}$, $Z_{\odot}$ models). This prediction might be measurable statistically. Finally, at central helium ignition the stellar radius and Ro decrease again. Ro remains of the order of unity while the stars stay on the clump and evolve on the early-AGB before increasing again as the star expands along the TP-AGB phase (not shown in Fig. 5, but see Fig. 6). Therefore, the models predict that the stars cross the magnetic strip twice during their post-main evolution, first on their way to the RGB, and second during central helium burning phase and the early-AGB.

\subsubsection{Mass dependence}

The general behavior of $\operatorname{Ro}\left(H_{p} / 2\right)$ along the evolution path of our models of different masses is shown in Fig. 6 for the grid at solar metallicity (the comparison with the observations is made in Sect. 5). The models with masses lower than or equal to $4 M_{\odot}$ behave like the $2 M_{\odot}$ case described above. For all of them, $\operatorname{Ro}\left(H_{p} / 2\right)$ (as well as $\mathrm{Ro}_{\max }$ and all $\mathrm{Ro}(\mathrm{r})$ ) drops below unity when the stars first cross the Hertzsprung gap and start ascending the RGB. The Rossby numbers then increase again until central helium burning ignites. In this region of the Hertzsprung-Russell diagram, a clear "magnetic strip" appears, where Rossby numbers consistently below unity are predicted and where an $\alpha-\Omega$ dynamo may thus be stronger. For higher mass stars the Rossby numbers do not reach such a low value in the post-MS phases, either because the stellar radius and the rotational period are higher $\left(Z_{\odot}\right.$ case), or because the stars ignite central helium-burning before they could ascend the RGB. We find the same behavior for all the metallicities.

For the stars with initial masses lower or equal to $4 M_{\odot}$, $\operatorname{Ro}\left(H_{p} / 2\right)$ (as well as all $\left.\operatorname{Ro}(r)\right)$ drops again below unity during central helium-burning, due to the decrease of the stellar radius. Rossby numbers rise again when the stars move towards the early-AGB phase. This second "magnetic strip" extends towards higher luminosity and more advanced evolution phases when the metallicity decreases.

In summary, the theoretical magnetic strips we just described appear to be well defined in terms of effective temperature range (i.e. for a given mass and metallicity this strip is well defined by using only the effective temperature; see Fig. 6). They comprise stars at the base of the RGB and in the core-helium burning phase. There appears to be an upper luminosity limit (around $\log L / L_{\odot} \sim 3$ ), as more massive stellar models (at $Z_{\odot}$ ) or models 

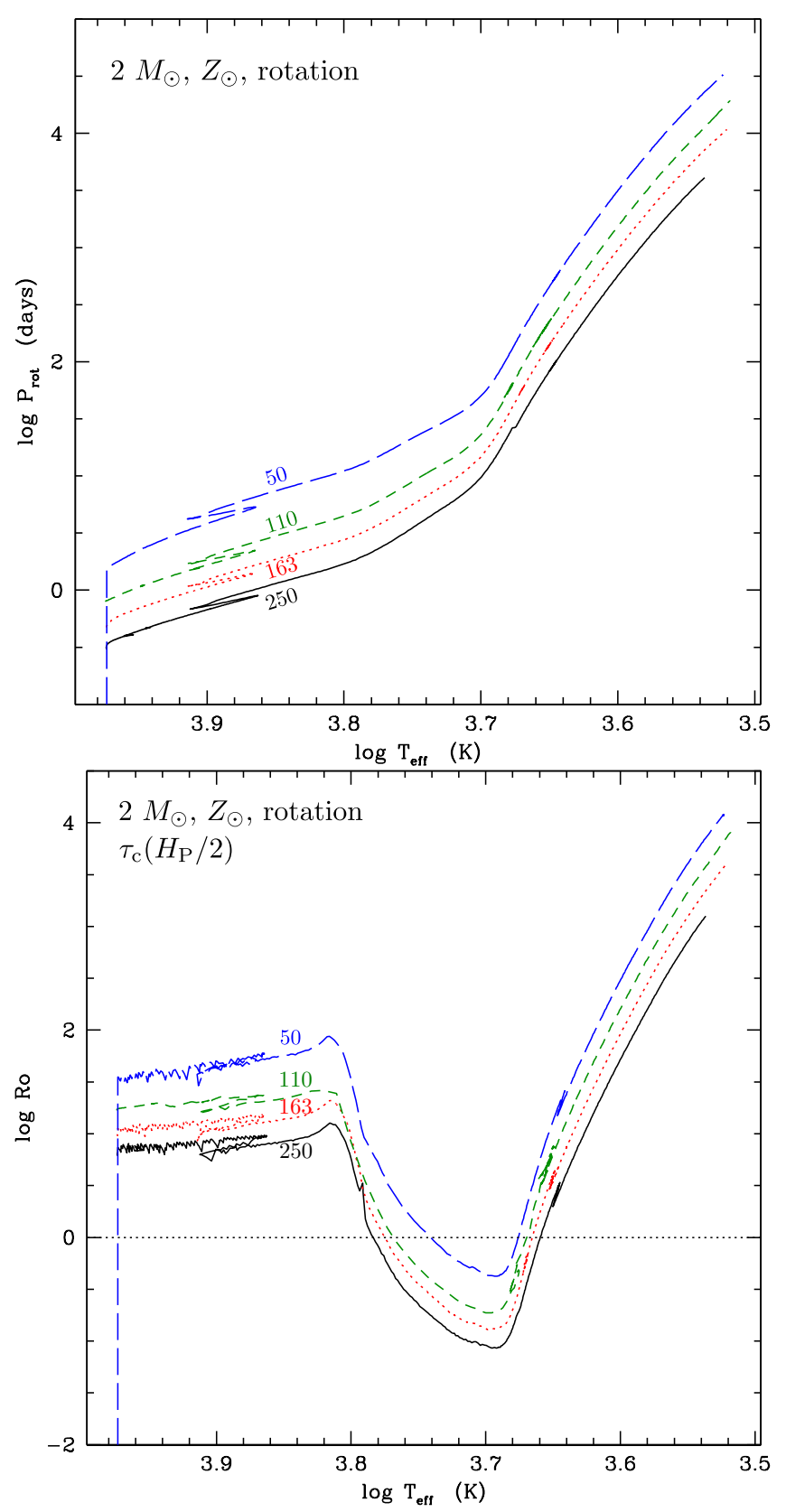

Fig. 5. Evolution of the rotation period (top) and Rossby number computed with the turnover timescale $\tau\left(H_{p} / 2\right)$ (bottom) from the ZAMS up to the RGB tip for the $2 M_{\odot}, Z_{\odot}$ models computed with different initial rotation velocities on the ZAMS $\left(50,110,163\right.$, and $\left.250 \mathrm{~km} \mathrm{~s}^{-1}\right)$. The dotted horizontal line indicates a Ro equal to 1 , which is the typical value to invoke $\alpha-\Omega$ dynamo.

of lower metallicity that exhibit blue loops during central helium burning do not predict Ro(r) decreasing below unity.

\subsubsection{Short note on the case of hot intermediate-mass stars}

Figure 6 reveals our most massive models predict $\operatorname{Ro}\left(H_{p} / 2\right)$ of the order of or lower than unity during some evolutionary phases at which the convective envelope is extremely thin (e.g., the $6 M_{\odot}, Z_{\odot}$ model on the MS, and the $6 M_{\odot}, Z=0.0001$ model from the ZAMS up to the end of central helium-burning). These low values of $\operatorname{Ro}\left(H_{p} / 2\right)$ are mainly due to the high rotation velocities of the models. However, the subsurface convective layers in these objects are caused by the helium opacity bump, and have
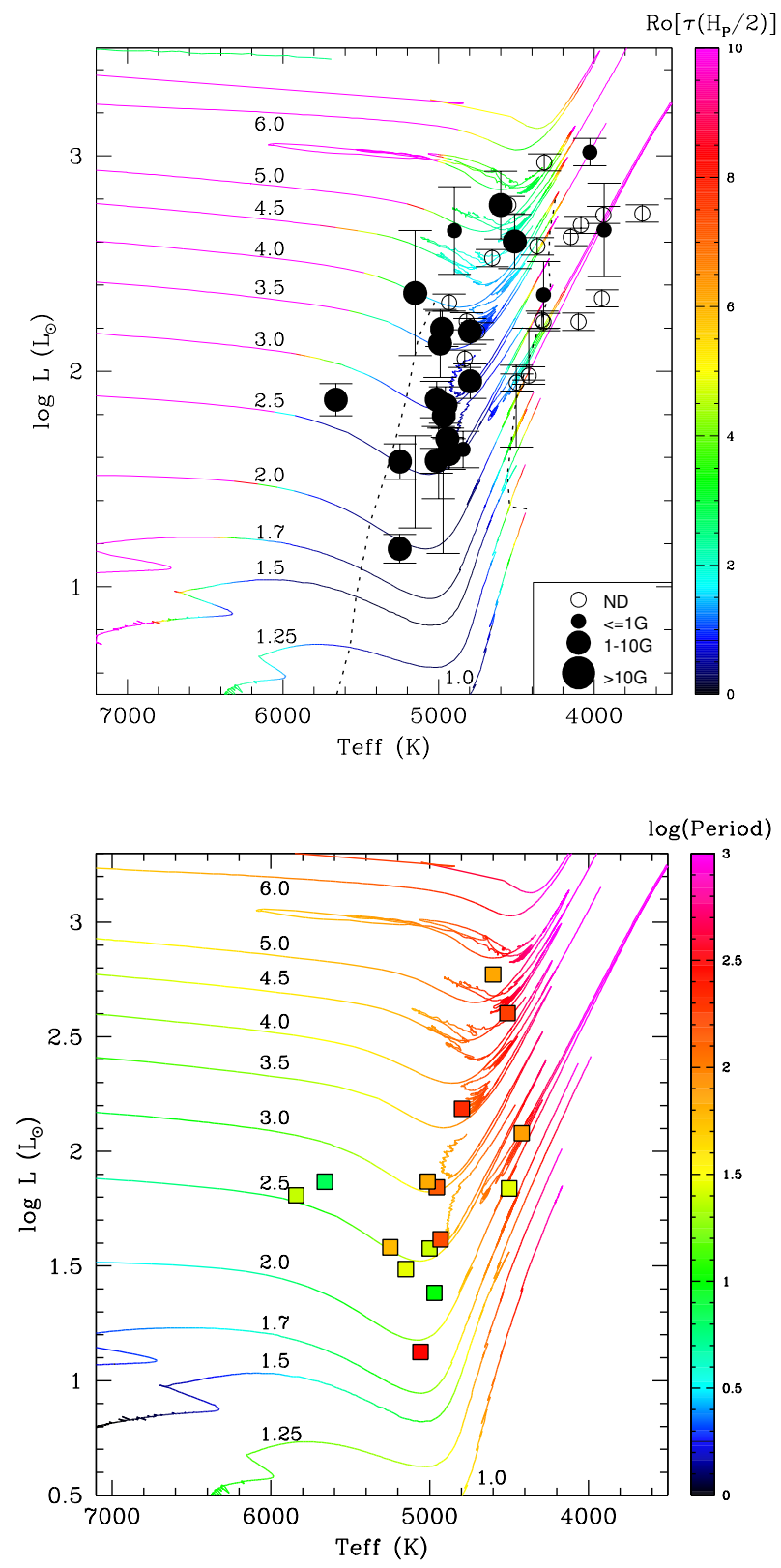

Fig. 6. Top: color-coded Rossby number $\operatorname{Ro}\left(H_{p} / 2\right)$ along the tracks in the HRD up to the RGB luminosity where $\operatorname{Ro}\left(H_{p} / 2\right)$ becomes higher than 10 (for stellar masses lower than $2.5 M_{\odot}$ ) or up to the early-AGB (for stellar masses higher than $2.5 M_{\odot}$ ), for the $Z_{\odot}$ models with rotation. Initial stellar masses are indicated on the tracks. Dotted lines indicate the beginning and end of the first dredge-up (when the mass of the convective envelope is $2.5 \%$ of the total stellar mass, left; at the maximum extent in mass of the convective envelope, right). The points correspond to the sample stars of Aurière et al. (2015); the size of the black symbols depicts the strength of the detected magnetic field, and open symbols stand for nondetected stars. Bottom: a color-coded logarithm scale is shown for the rotation period (in days) along the tracks and for the sample stars of Aurière et al. (2015) that have known rotation periods (squares).

a very limited extension (Fig. 4 , where all $Z_{\odot}$ models with initial mass higher than $2 M_{\odot}$ have convective layers that are thinner than one pressure scale height); there, convection is very inefficient and transports only a small fraction of the heat flux. As a consequence, these stars are not expected to develop $\alpha-\Omega$ dynamo, despite low theoretical Rossby numbers. 


\section{Magnetic strips for evolved low- and intermediate-mass stars - comparison with observations}

We compare in Fig. 6 our model predictions for $\operatorname{Ro}\left(H_{p} / 2\right)$ along the evolution tracks with the positions in the HRD of the spectropolarimetric sample of evolved stars studied by Aurière et al. (2015). We exclude the stars that are probable Ap descendants, and keep only the stars where the Zeeman effect was detected and which are believed to have $\alpha-\Omega$ dynamos. We also show the sample stars that have no magnetic field detected. While the latest ones were chosen through different selection criteria than the other sample stars, they are clearly not active at the level of the $\alpha-\Omega$ dynamo stars detected in the magnetic strip (that is, below $\sim 1 \mathrm{G}$; see Aurière et al. 2015 for details). Figure 6 clearly shows that the vast majority of Zeeman detected evolved stars lie in the area along the tracks where $\operatorname{Ro}\left(H_{p} / 2\right)$ is close to minimum values around or below unity, while most of the non-magnetic stars lie outside the theoretical magnetic strips (that is, the areas where $\operatorname{Ro}\left(H_{p} / 2\right)$ becomes much larger than unity). The only exceptions are the couple of very bright, very cool giant stars with weak detected magnetic fields (below $1 \mathrm{G}$ ) for which we predict a Rossby number of the order of 10; unfortunately, the rotation period of these two stars is unknown, but they have very low Vsini, indicating that the $\alpha-\Omega$ dynamo is unlikely to operate in these objects.

We remind that the values of $\mathrm{Ro}_{\max }$ (that is, computed with $\left.\tau_{\max }\right)$ along individual tracks are lower than the values of $\operatorname{Ro}\left(H_{p} / 2\right)$. Since it is not well established yet where the dynamo operates in the convective envelope of cool evolved stars, the agreement seen in Fig. 6 between the theoretical and observational magnetic strips appears thus to be very satisfactory. This is remarkable especially given that our stellar models are computed for only one initial rotation rate $(30 \%$ of the critical rotation velocity on the ZAMS). However and as can be seen in Fig. 6, the observations reveal a spread in rotation periods for stars that lie close to one another in the Hertzsprung gap, which probably reflects the fact that the sample stars may have started their life with a range of initial rotation velocities. This effect can be estimated from Fig. 5, where we show the evolution of the theoretical rotation period for the $2 M_{\odot}, Z_{\odot}$ model computed with different initial rotation rates. It is dominated by the fast secular increase of the stellar radius that occurs much faster than the internal transport of angular momentum when the star is crossing the Hertzsprung gap. At the effective temperature of one of the lowest-mass stars observed by Aurière et al. (2015, HD 203387; $T_{\text {eff }}=5012 \mathrm{~K}, \log L / L_{\odot}=1.87$ ) the theoretical period of the $2 M_{\odot}$ model varies between 10 and 60 days (which implies a factor of 6 variation on the theoretical Rossby between the two models) depending on the initial rotation velocity, while the observed period for this star is 68 days. While the overall agreement is relatively good, comparison with the magnetic and rotational properties of individual stars requires tailor-made models with different initial rotation rates for all individual sample stars (e.g., Aurière et al. 2009, 2011, 2012; Konstantinova-Antova et al. 2010, 2012; Tsvetkova et al. 2013; Borisova et al. 2016; Tsvetkova et al. 2017), which is out of the scope of the present paper.

According to our models, the magnetic strips we observe and predict for intermediate-mass stars should extend to the low-mass, low-luminosity regime. Low-mass stars (below $2 M_{\odot}$ ) should thus also undergo $\alpha-\Omega$ dynamo mechanisms while they cross the Hertzsprung gap and reach the base of the $\mathrm{RGB}^{3}$. This could easily be tested observationally, although to the best of our knowledge, there is no magnetic field detection in low-mass (that is, below $1.5 M_{\odot}$ ) RGB stars quoted in the literature.

\section{Conclusions}

Both quantities used to compute the Rossby number, that is, convective and rotational velocities, depend sensitively on the stellar mass, metallicity, and evolutionary phase. Here we use stellar evolution models that include rotation (Lagarde et al. 2012) to predict these quantities and their temporal variation due to stellar evolution for a large range of initial masses and metallicities. We compute convective turnover times and Rossby numbers at different depths in the stellar convective envelope. Although the predicted absolute values for these quantities depend on model input physics, such as $\alpha_{\text {MLT }}$ (e.g., Kim \& Demarque 1996) and initial rotation rate, the evolutionary (temporal) trends are expected to be largely insensitive to such assumptions. Thus, the predicted tendencies for small Rossby numbers during certain evolutionary phases should be robust, providing a likely explanation of recent magnetic field detections in giant stars in terms of an $\alpha-\Omega$ dynamo mechanism analogous to solar-like stars.

Our models reveal sharp increases in turnover timescales and decreases in rotation rate that are associated to secular evolution effects. This translates into sharp decreases in Rossby number at certain evolutionary phases. These phases show up as welldelineated regions in the HRD that correspond to the first dredgeup phase at the base of the RGB, and to core-helium burning and early AGB. The predicted low Rossby numbers suggest that $\alpha-\Omega$ dynamos may operate in the convective envelopes of low- and intermediate-mass stars during these phases. This offers an explanation for the observed magnetic strips in the HRD that are populated by evolved intermediate-mass stars exhibiting strong magnetic features (Konstantinova-Antova et al. 2013; Aurière et al. 2015). Our prediction that post-main sequence low-mass stars also cross the magnetic strips could be easily tested in the near future with dedicated spectro-polarimetric observations. Our models also predict that the width (in effective temperature) of the magnetic strips depends on the initial rotation rate. Thus, the dispersion of $T_{\text {eff }}$ among magnetic giants with fast rotation should be statistically larger than that among slower rotators. This could in principle be tested for a statistically significant sample of evolved stars with magnetic detection and rotation rate determination. Finally, these predictions provide guidance for future magnetohydrodynamic simulations of global stellar dynamos aimed at understanding the precise locus of magnetic field generation in the outer convective layers of evolved stars.

Acknowledgements. The authors acknowledge financial support from the Swiss National Science Foundation (SNSF) and the French Programme National de Physique Stellaire (PNPS) of CNRS/INSU. T.D. acknowledges financial support from the UE Programme (FP7/2007-2013) under grant agr. No. 267251 "Astronomy Fellowships in Italy" (ASTROFit). N.L. acknowledges financial support from Marie Curie Intra-european fellowship (FP7-PEOPLE-2012-IEF) and from the CNES fellowship. R.K.-A. acknowledges the support of the BulgarianFrench program RILA-CAMPUS, project DRILA 01/3. F.G. acknowledges financial support from COST Action TD 1308. S.M. acknowledges funding by the European Research Council through ERC grant SPIRE 647383. We thank the anonymous referee for constructive comments that helped improving the presentation of our results.

\footnotetext{
3 We can not compute the theoretical Rossby numbers at the clump and on the AGB for the low-mass models of Lagarde et al. (2012), since they do not follow rotation beyond the RGB tip.
} 


\section{References}

Alecian, E., Wade, G. A., Catala, C., et al. 2013, MNRAS, 429, 1001 Amard, L., Palacios, A., Charbonnel, C., Gallet, F., \& Bouvier, J. 2016, A\&A 587, A 105

Amard, L., Palacios, A., Mathis, S., et al. 2017, A\&A, submitted

Asplund, M., Grevesse, N., \& Sauval, A. J. 2005, in Cosmic Abundances as Records of Stellar Evolution and Nucleosynthesis, eds. T. G. Barnes III, \& F. N. Bash, ASP Conf. Ser., 336, 25

Augustson, K. C., Brun, A. S., \& Toomre, J. 2016, ApJ, 829, 92

Augustson, K., Mathis, S., \& Brun, A. S. 2017, ArXiv e-prints [arXiv: 1701.02582]

Aurière, M., Wade, G. A., Konstantinova-Antova, R., et al. 2009, A\&A, 504, 231 Aurière, M., Konstantinova-Antova, R., Petit, P., et al. 2011, A\&A, 534, A139

Aurière, M., Konstantinova-Antova, R., Petit, P., et al. 2012, A\&A, 543, A118 Aurière, M., Lignières, F., Konstantinova-Antova, R., et al. 2014, in Putting A Stars into Context: Evolution, Environment, and Related Stars, eds. G. Mathys, E. R. Griffin, O. Kochukhov, R. Monier, \& G. M. Wahlgren, 444 Aurière, M., Konstantinova-Antova, R., Charbonnel, C., et al. 2015, A\&A, 574, A90

Beck, P. G., Montalban, J., Kallinger, T., et al. 2012, Nature, 481, 55

Borisova, A., Aurière, M., Petit, P., et al. 2016, A\&A, 591, A57

Brown, B. P., Browning, M. K., Brun, A. S., Miesch, M. S., \& Toomre, J. 2008 ApJ, 689, 1354

Brun, A. S. 2015, IAU General Assembly, 22, 2244193

Brun, A. S., Browning, M. K., \& Toomre, J. 2005, ApJ, 629, 461

Brun, A. S., Browning, M. K., Dikpati, M., Hotta, H., \& Strugarek, A. 2015 Space Sci. Rev., 196, 101

Cantiello, M., Mankovich, C., Bildsten, L., Christensen-Dalsgaard, J., \& Paxton, B. 2014, ApJ, 788, 93

Ceillier, T., Eggenberger, P., García, R. A., \& Mathis, S. 2013, A\&A, 555, A54

Charbonneau, P. 2014, ARA\&A, 52, 251

Charbonneau, P., \& MacGregor, K. B. 1993, ApJ, 417, 762

Charbonnel, C., \& Lagarde, N. 2010, A\&A, 522, A10

Cunha, K., Hubeny, I., \& Lanz, T. 2006, ApJ, 647, L143

Decressin, T., Mathis, S., Palacios, A., et al. 2009, A\&A, 495, 271

Deheuvels, S., García, R. A., Chaplin, W. J., et al. 2012, ApJ, 756, 19

Deheuvels, S., Doğan, G., Goupil, M. J., et al. 2014, A\&A, 564, A27

Denissenkov, P. A., Pinsonneault, M., Terndrup, D. M., \& Newsham, G. 2010, ApJ, 716, 1269

Di Mauro, M. P., Ventura, R., Cardini, D., et al. 2016, ApJ, 817, 65

Donati, J.-F., \& Landstreet, J. D. 2009, ARA\&A, 47, 333

Durney, B. R., \& Latour, J. 1978, Geophysical and Astrophysical Fluid Dynamics, 9, 241

Eggenberger, P., Maeder, A., \& Meynet, G. 2005, A\&A, 440, L9

Eggenberger, P., Montalbán, J., \& Miglio, A. 2012, A\&A, 544, L4

Folsom, C. P., Petit, P., Bouvier, J., et al. 2016, MNRAS, 457, 580

Gilliland, R. L. 1985, ApJ, 299, 286

Gough, D. O., \& McIntyre, M. E. 1998, Nature, 394, 755

Gregory, S. G., Donati, J.-F., Morin, J., et al. 2012, ApJ, 755, 97
Kawaler, S. D. 1988, ApJ, 333, 236

Kim, Y.-C. \& Demarque, P. 1996, ApJ, 457, 340

Kippenhahn, R., Weigert, A., \& Weiss, A. 2012, Stellar Structure and Evolution (Berlin, Heidelberg: Springer-Verlag)

Konstantinova-Antova, R., Aurière, M., Charbonnel, C., et al. 2010, A\&A, 524, A57

Konstantinova-Antova, R., Aurière, M., Petit, P., et al. 2012, A\&A, 541, A44

Konstantinova-Antova, R., Aurière, M., Charbonnel, C., et al. 2013, Bulg. Astron. J., 19, 14

Lagarde, N., Charbonnel, C., Decressin, T., \& Hagelberg, J. 2011, A\&A, 536, A28

Lagarde, N., Decressin, T., Charbonnel, C., et al. 2012, A\&A, 543, A108

Lagarde, N., Anderson, R. I., Charbonnel, C., et al. 2014, A\&A, 570, C2

Landin, N. R., Mendes, L. T. S., \& Vaz, L. P. R. 2010, A\&A, 510, A46

Maeder, A., \& Zahn, J.-P. 1998, A\&A, 334, 1000

Mangeney, A., \& Praderie, F. 1984, A\&A, 130, 143

Marques, J. P., Goupil, M. J., Lebreton, Y., et al. 2013, A\&A, 549, A74

Mathis, S., \& Zahn, J.-P. 2004, A\&A, 425, 229

Mathis, S., \& Zahn, J.-P. 2005, A\&A, 440, 653

Mathis, S., Prat, V., Amard, L., et al. 2017a, A\&A, submitted

Mathis, S., Amard, L., Charbonnel, C., et al. 2017b, A\&A, submitted

Matt, S. P., Brun, A. S., Baraffe, I., Bouvier, J., \& Chabrier, G. 2015, ApJ, 799, L23

Mosser, B., Goupil, M. J., Belkacem, K., et al. 2012a, A\&A, 548, A10

Mosser, B., Goupil, M. J., Belkacem, K., et al. 2012b, A\&A, 540, A143

Neiner, C., Mathis, S., Alecian, E., et al. 2015, IAU Symp., 305, 61

Noyes, R. W., Hartmann, L. W., Baliunas, S. L., Duncan, D. K., \& Vaughan, A. H. 1984, ApJ, 279, 763

Palacios, A., Talon, S., Charbonnel, C., \& Forestini, M. 2003, A\&A, 399, 603

Palacios, A., Charbonnel, C., Talon, S., \& Siess, L. 2006, A\&A, 453, 261

Réville, V., Brun, A. S., Strugarek, A., et al. 2015, ApJ, 814, 99

Rossby, C. G. 1939, J. Marine Res., 2, 38

Rucinski, S. M., \& Vandenberg, D. A. 1990, AJ, 99, 1279

Schatzman, E. 1962, Annales d'Astrophysique, 25, 18

Spruit, H. C. 1999, A\&A, 349, 189

Stello, D., Cantiello, M., Fuller, J., et al. 2016, Nature, 529, 364

Strugarek, A., Brun, A. S., \& Zahn, J.-P. 2011, A\&A, 532, A34

Suijs, M. P. L., Langer, N., Poelarends, A.-J., et al. 2008, A\&A, 481, L87

Talon, S., \& Charbonnel, C. 2003, A\&A, 405, 1025

Talon, S., \& Charbonnel, C. 2008, A\&A, 482, 597

Talon, S., \& Zahn, J.-P. 1998, A\&A, 329, 315

Tsvetkova, S., Petit, P., Aurière, M., et al. 2013, A\&A, 556, A43

Tsvetkova, S., Petit, P., Konstantinova-Antova, R., et al. 2017, A\&A, 599, A72

Turck-Chièze, S., Palacios, A., Marques, J. P., \& Nghiem, P. A. P. 2010, ApJ, 715, 1539

Varela, J., Strugarek, A., \& Brun, A. S. 2016, Adv. Space Res., 58, 1507

Vidotto, A. A., Gregory, S. G., Jardine, M., et al. 2014, MNRAS, 441, 2361

Wade, G. A., Neiner, C., Alecian, E., et al. 2016, MNRAS, 456, 2

Zahn, J.-P. 1992, A\&A, 265, 115 


\section{Appendix A: Turnover timescale and Rossby number in stellar convective cores along the main sequence}

This paper focusses on possible development of $\alpha-\Omega$ dynamo along the so-called magnetic strips in the advanced phases of stellar evolution (Konstantinova-Antova et al. 2013; Aurière et al. 2015) when stars have extended convective envelopes. However, internal magnetic fields might also be produced in intermediate-mass main sequence stars that host convective cores (e.g., Brun et al. 2005; Stello et al. 2016). Therefore, we have computed the turnover timescales and Rossby numbers in stellar convective cores along the main sequence for $Z_{\odot}$ models with masses between 2 and $6 M_{\odot}$. We show in Fig. A.1 the global turnover timescale for the entire core and the associated Rossby number. As for the case of the convective envelope, the values we get for the turnover timescale in the convective core are not affected by rotation, and their quantitative estimate is solid (within the MLT framework). For all the models $\tau_{\mathrm{g}}$ decreases in the core along the MS (for the $2 M_{\odot}, Z_{\odot}$ model, $\tau_{\mathrm{g}}$ is very similar in the convective core and the thin convective envelope; compare Fig. 3). On the other hand, the rotation rate of the convective core increases along the MS for all the models because of secular evolution effects. The Rossby number of the core thus also slightly increases with time, and this evolution trend is a robust prediction. For the present models, the Rossby number of the core remains well below unity all along the MS. This results from the relatively weak coupling between the central and the external regions in the models of Lagarde et al. (2012), due to the adopted assumptions for the transport of angular momentum by turbulence and meridional circulation. However, the theoretical core rotation rates are overestimated compared to asteroseismic constraints, which is a well known problem for all the current models including rotation (Talon \& Zahn 1998; Turck-Chièze et al. 2010; Denissenkov et al. 2010; Eggenberger et al. 2012; Beck et al. 2012; Deheuvels et al. 2012, 2014; Mosser et al. 2012a; Marques et al. 2013; Ceillier et al. 2013; Di Mauro et al. 2016). Therefore, the values we derive for the central Rossby number are certainly underestimated. A consistent study of the dynamo in convective stellar cores would thus require models including very efficient transport of angular momentum in stellar interiors, with processes related, for example, to internal gravity waves (Talon \& Charbonnel 2003, 2008), to fossil magnetic fields (Charbonneau \& MacGregor 1993; Gough \& McIntyre 1998; Eggenberger et al. 2005; Strugarek et al. 2011), or including new prescriptions for rotation-induced turbulence proposed by Mathis \& et al. (2017a) that lead to better agreement with asteroseismology (Mathis \& et al. 2017b; Amard et al. 2017). This is out of the scope of the present paper.
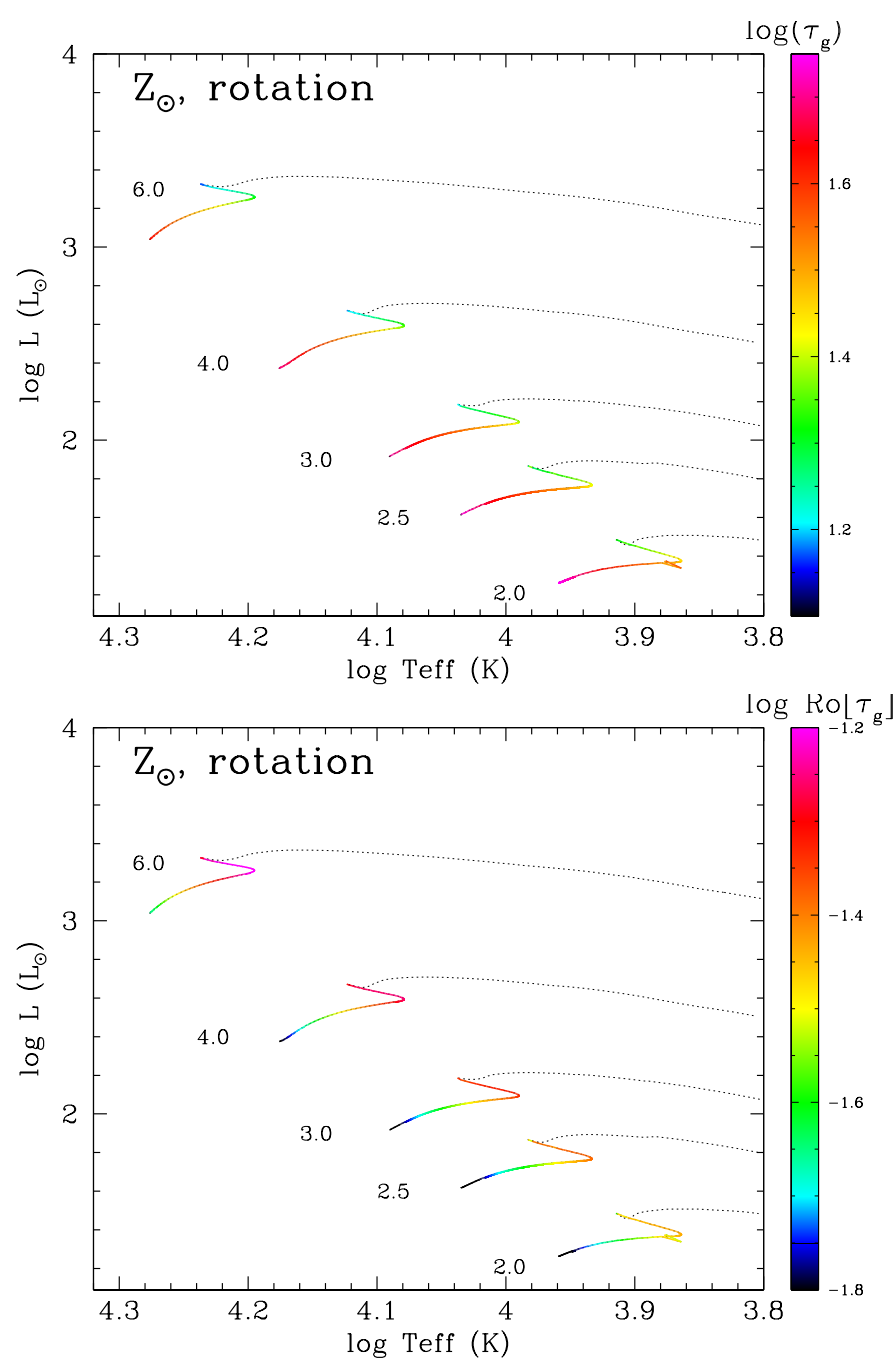

Fig. A.1. Color-coded global convective turnover time $\tau_{\mathrm{g}}$ and corresponding Rossby number (in logarithmic scale) in the stellar convective core along the MS for the solar metallicity models. Initial stellar masses (in solar mass) are indicated on the tracks. 